$(60.6 \%$ vs $36.7 \%)$ and those without evidence of disease at 12 months post transplantation $(67.5 \%$ vs $5.7 \%)$ had higher survival. In multivariate analysis, evidence of disease at 12 months after HSCT (HR 5.22), chemo-sensitivity to the last regimen (HR 6.81) and systemic symptoms (HR 2.60) were associated to survival. Conclusions We found that the most well recognised disease characteristics and overall survival in this cohort were similar to those found for patients with NHL undergoing haematopoietic stem cell transplantation in other countries.

\section{SP1-42 PREVALENCE OF OVERWEIGHT AND ASSOCIATED FACTORS IN UNDER-5-YEAR-OLD CHILDREN IN BRAZIL}

doi:10.1136/jech.2011.142976n.19

${ }^{1} \mathrm{R}$ Müller, ${ }^{2} \mathrm{E}$ Tomasi, ${ }^{2} \mathrm{~L}$ Facchini, ${ }^{2} \mathrm{R}$ Piccini, ${ }^{2} \mathrm{D}$ Silveira, ${ }^{2} \mathrm{~F}$ Siqueira, ${ }^{2} \mathrm{E}$ Thumé, ${ }^{2} \mathrm{~S}$ Silva, ${ }^{2} \mathrm{~A}$ Dilélio. ${ }^{1}$ Universidade Católica de Pelotas, Pelotas, Rio Grande do Sul, Brazil; ' Universidade Federal de Pelotas, Pelotas, Rio Grande do Sul, Brazil

Introduction The aim of the study was to determine the prevalence of overweight among under-5-year-old children in Brazil and investigate its associations with sociodemographic characteristics, exclusive breastfeeding, number of siblings and birth weight.

Methods Cross sectional population based study, conducted in the five geopolitical regions of Brazil, with a sample of 6397 children. The nutritional classification was done using the 2006 WHO growth curves. Were considered overweight the children with a zscore higher than two SDs above the weight for height median.

Results The prevalence of overweight among under-5-year-old children in Brazil was $12 \%$. The outcome was $22 \%$ higher in males $(\mathrm{RP}=1.22 ; 95 \% \mathrm{CI} 1.02$ to $1.47 ; \mathrm{p}=0.030)$. There was a linear inverse association: the younger the child, the higher the prevalence of overweight $(p=0.032)$. The white children had a prevalence of overweight $22 \%$ higher than the non-white ones. The higher the birth weight, the higher the prevalences of overweight $(p=0.000)$. Children who were breastfed up to 120 days had a prevalence of overweight $34 \%$ higher compared to the ones who were breastfed for more than 120 days.

Conclusion The prevalence of obesity was higher in males, in under1 -year-old, white, with a birth weight of $<3500 \mathrm{~g}$, exclusively breastfed up to 120 days children.

\section{SP1-43 CLASS-BASED RESIDENTIAL SEGREGATION AND SOCIOECONOMIC DISPARITIES IN ASTHMA PREVALENCE}

doi:10.1136/jech.2011.142976n.20

K Kershaw, ${ }^{*}$ M Carnethon. Northwestern University, Chicago, Illinois, USA

Socioeconomic disparities in asthma prevalence are well established in the US. Evidence suggests environmental factors may play a role, but no studies have examined the role of class-based residential segregation. We investigated whether class-based residential segregation attenuated the association between individual-level income and asthma prevalence among 164143 non-Hispanic (NH) white, $19493 \mathrm{NH}$ black, and 14399 Hispanic participants of the 2009 Behavioural Risk Factor Surveillance System aged 18 years and older. Current asthma was based on self-report. Class-based segregation was measured at the metropolitan level using the poverty isolation index, a measure of the extent to which individuals with incomes below the poverty threshold are spatially isolated from non-poor individuals. Each metropolitan area was given an index score ranging from near 0 to 1 , with lower scores indicating less segregation. Among blacks, odds of asthma was 0.84 times lower per quartile higher income ( $95 \%$ CI 0.77 to 0.91 ) after adjusting for age and gender. However, this association varied by level of segregation ( $p$ for interaction=0.07). Income was more weakly associated with odds of current asthma at low segregation (10th percentile; OR $0.90 ; 95 \%$ CI 0.78 to 1.03 ) vs high segregation (90th percentile; OR $0.74 ; 95 \%$ CI 0.66 to 0.82 ). For whites, income was also inversely associated with odds of current asthma, but adjustment for segregation did not attenuate this relationship. Neither income nor segregation was associated with current asthma among Hispanics. These findings suggest that among blacks, class-based segregation may help explain individual-level income disparities in asthma prevalence.

\section{SP1-44 ASSOCIATION BETWEEN ALLERGIC DISEASES AND NUTRITIONAL STATUS AMONG CHILDREN IN BANGLADESH}

doi:10.1136/jech.2011.142976n.21

${ }^{1} \mathrm{H}$ M D Hossain, ${ }^{*}{ }^{1}$ E Noguchi, ${ }^{2} \mathrm{~S}$ E Arifeen, ${ }^{2} \mathrm{R}$ Raqib, ${ }^{3} \mathrm{~L}$ A Persson, ${ }^{1} \mathrm{Y}$ Wagatsuma. ${ }^{1}$ Department of Epidemiology, University of Tsukuba, Tsukuba, Japan; ${ }^{2}$ International Centre for Diarrhoeal Diseases Research, Bangladesh (ICDDR,B), Dhaka, Bangladesh; ${ }^{3}$ Department of International Women and Child Health, Uppsala University, Uppsala, Sweden

Introduction Interest has been arisen whether nutritional status is related to development of allergic diseases in children. Our aim was to investigate the association between nutritional status and serum IgE level in the developing country.

Methods This cross-sectional study was nested into a large scale nutrition intervention trial among pregnant women in rural Bangladesh. In this follow-up study, we collected venous blood to measure serum total and specific IgE. Serum total IgE was measured by human IgE quantitative ELISA. And IgE specific to dust-mite and ascaris were measured by the CAP-FEIA system. Weight and height have been measured and stunting, wasting, under weight and overweight were calculated by WHO Anthro. Specific IgE $>0.70 \mathrm{UA} / \mathrm{ml}$ was considered as positive.

Results A total of 912 children of 4.5 years of age was successfully completed the study. Anthropometric indicators revealed wasting in $17 \%$, stunting in $32 \%$, underweight in $41 \%$ and overweight in $0.2 \%$ of the children. Log total IgE was $2.69+0.27 \mathrm{IU} / \mathrm{ml}$ (mean+SD). Mean anti-DP specific IgE was 3.33 (range: $0.00->100$ ) UA/ml. Mean anti-ascaris specific IgE was 11.89 (range: $0.00->100$ ) UA/ml. Stunting was significantly associated with increased total IgE (OR (95\% CI) 1.59 (1.01 to 2.50)) and anti-ascaris IgE (OR (95\% CI) 1.65 (1.18 to 2.29$)$ ). The association remained statistically significant after adjustment for mother's BMI, sex, health status and current illnesses ( $p=0.044$ and $p=0.003$ respectively).

Conclusion The total and specific IgE level was high among children in Bangladesh. Nutritional status had an association with increased total and anti-ascaris IgE antibody.

\section{SP1-45 PREVALENCE OF DEPRESSION IN PATIENTS WITH IDIOPATHIC PARKINSON'S DISEASE IN KOREA}

doi:10.1136/jech.2011.142976n.22

W C Kim, ${ }^{*}$ H S Kim, S H Oh, O J Kim. Department of Neurology, CHA Bundang Medical Center, Seongnam, Gyeonggi-do, Republic of Korea

Introduction Depression is one of the most common non-motor symptoms of Parkinson's disease (PD). The prevalence rates vary widely according to the diagnostic criteria. However, in Korea, there are very few epidemiologic data concerning the prevalence of depression in PD. The aim of this study is to investigate the prevalence of depression and factors influencing depression in patients with PD. 
Methods Newly diagnosed 252 consecutive PD patients were included and followed as part of an ongoing PD registry. PD was diagnosed according to the United Kingdom brain bank diagnostic criteria. 79 PD patients fulfilled the DSM-IV criteria for major depression. The UPDRS motor score was checked at the best "on" period to assess the clinical severity of $\mathrm{PD}$. We compared the clinical data between depressive (DP, $\mathrm{n}=79$ ) and non-depressive (NDP, $\mathrm{n}=173$ ) groups.

Results The prevalence rate of depression in $\mathrm{PD}$ was $31.3 \%$ in this study. There was no difference in age (DP: $62.3 \pm 2.5$, NDP: $59.8 \pm 2.7 \mathrm{yrs}$ ), age of disease onset (DP: $52.0 \pm 3.7 \mathrm{yrs}$, NDP: $54.7 \pm 3.8 \mathrm{yrs}$ ), UPDRS motor scores (DP: $36.2 \pm 5.6$, NDP: $33.8 \pm 3.7$ ) and Hoehn and Yahr stage (DP: $3.34 \pm 0.52$, NDP: 2.91 \pm 0.63 ) between two groups.

Conclusion There was no significant difference in clinical features between DP and NDP groups in this study. These results suggest that depression in $\mathrm{PD}$ is not influenced the severity of motor symptoms and that non-dopaminergic neurotransmitters, such as norepinephrine and acetylcholine, at least associated with the pathophysiology of depression in PD.

\section{SP1-46 SCHOOL-BASED INTERVENTION TO PROMOTE HEALTHY NUTRITION AND PHYSICAL ACTIVITY IN SOUSSE, TUNISIA}

doi:10.1136/jech.2011.142976n.23

M M Jihene, ${ }^{*}$ H Imed, G Rafika, B A Imene, B Mylene, G Hassen. Farhat Hached Hospital, Sousse, Tunisia

Throughout the world, and particularly in Tunisia, children are becoming overweight and obese at progressively younger ages. Our purpose was to evaluate the effects of a school intervention program to promote healthy nutrition and physical activity among adolescents, in terms of behaviours and intention.

Methods It was a quasi experimental intervention study with two groups: control and intervention group with pre-post evaluation of nutrition and physical activity intention and behaviour in each group. The target population was composed with students aged $12-16$ years schooled in colleges of Sousse in Tunisia. To evaluate the intervention, a sampling was used to include 2200 students who participated to the questionnaire. All the students of intervention group received a standardised program with information about healthy nutrition and physical activity. An Arabic pre-tested and auto-administered questionnaire was used to assess nutrition and physical activity intention and behaviour before and after the intervention.

Results The intervention group's posttest intention and behaviour were significantly higher than the control group's posttest. Concerning "healthy behaviours", in the intervention group, children improved significantly their vegetable intake. It passed from $45.4 \%$ to $56.8 \%(\mathrm{p}<0.001)$. The proportion of children who practice 5 days per week physical activity for at least $30 \mathrm{~min}$ a day increased significantly from $23 \%$ to $44 \%$ ( $p<0.001)$.

Conclusion School based intervention to promote healthy diet and physical activity is benefit and important at the middle age to prevent obesity and cardiovascular disease risk factors.

\section{SP1-47 ASSOCIATIONS BETWEEN PERSISTENT ORGANIC POLLUTANTS (POPS) IN VISCERAL AND SUBCUTANEOUS ADIPOSE TISSUE AND INSULIN RESISTANCE}

doi:10.1136/jech.2011.142976n.24

${ }^{1} Y$ M Lee, ${ }^{*}{ }^{1} S$ A Kim, ${ }^{2}$ S G Kim, ${ }^{3}$ K K Lee, ${ }^{1}$ B Y Chun, ${ }^{1} \mathrm{D}$ H Lee. ${ }^{1}$ Department of Preventive Medicine, School of Medicine, Kyungpook National University, Daegu, Republic of Korea; ' 2 Department of Surgery, School of Medicine, Kyungpook National
University, Daegu, Republic of Korea; ${ }^{3}$ Department of Internal Medicine, School of Medicine, Kyungpook National University, Daegu, Republic of Korea

Introduction Persistent Organic Pollutants (POPs) is recently linked to insulin resistance and type 2 diabetes. Although POPs are mostly bioaccumulated in adipose tissues, most studies have measured serum concentration of POPs because of difficulties of collecting adipose tissues. This study was performed 1) to compare patterns of concentrations of POPs between visceral adipose tissue (VAT) and subcutaneous adipose tissue (SAT), and 2) to investigate associations of insulin resistance with concentrations of POPs in VAT or SAT.

Methods We collected both VAT and SAT from 50 patients who underwent abdominal surgery and analysed 14 organochlorinated pesticides (OCPs) and 19 Polychlorinated biphenyls (PCBs). Insulin resistance was estimated using homeostasis model assessment method (HOMA-IR).

Results Concentrations of OCPs and PCBs among VAT and SAT were highly correlated, but absolute concentrations of PCBs in VAT were 3-4 times higher than those of SAT. As concentrations of p,p'-DDT, p,p'-DDD, cis-nonachlordane, trans-nonachlordane, PCB28, PCB105, and PCB118 in VAT or SAT increased, HOMA-IR significantly increased. The risk of elevated HOMA-IR $(>50$ th percentile) was 5 to 10 times higher among subjects in the 3rd tertile of these POPs compared with those in the 1st tertile. Although here are some differences depending on individual POP, the positive associations between POPs and HOMA-IR were generally more obvious in VAT than SAT. Also, the extent of macrophage infiltration in VAT was positively associated with concentrations of POPs in VAT, not SAT.

Conclusion The current study strongly suggested that some POPs accumulated in VAT may be involved in the development of insulin resistance.

\section{SP1-48 REDUCTIONS IN DENTAL CARIES IN 3-YEAR-0LD CHILDREN IN GREATER GLASGOW AND CLYDE}

doi:10.1136/jech.2011.142976n.25

${ }^{1}$ A D McMahon, * ${ }^{2} Y$ Blair, ${ }^{2} \mathrm{D}$ R McCall, ${ }^{1} \mathrm{~L}$ M D Macpherson. ${ }^{1}$ Glasgow University Dental School, Glasgow, Scotland, UK; ${ }^{2}$ NHS Greater Glasgow and Clyde, Glasgow, Scotland, UK

The objective of this study was to examine dental inspection data from 3-year-old children in Greater Glasgow and Clyde, Scotland, over 4 years to assess oral health trends during a period of implementation of a national child dental health improvement programme (Childsmile). Dental inspections of 3-year-old children in Greater Glasgow and Clyde were undertaken in the academic years 2006/7, 2007/8 (the baseline years), 2008/9 and 2009/10 (post intervention). The number of decayed, missing and filled teeth was calculated $\left(\mathrm{d}_{3} \mathrm{mft}\right)$, together with the percentage $\mathrm{d}_{3} \mathrm{mft}>0$. The study also examined the effect of socioeconomic status using the Scottish Index of Multiple Deprivation (SIMD). In total, 10022 children were inspected (19\% of the population). The weighted percentage of children with caries experience was $26 \%$ in $2006 / 7,25 \%$ (2007/8), reducing to $18 \%$ (2007/8) and 17\% (2009/ 10). When compared to the first baseline year of $2006 / 7$, the OR was 0.91 for 2007/8 (0.79-1.06, $p=0.221), 0.63$ for 2008/9 $(0.55-0.72, \mathrm{p}<0.001)$, and 0.50 for $2009 / 10(0.43-0.58, \mathrm{p}<0.001)$. The weighted mean $\mathrm{d}_{3} \mathrm{mft}$ was 1.1 in $2006 / 7,1.0$ in $2007 / 8$ $(p=0.869), 0.6$ in $2008 / 9(p<0.001)$ and 0.4 in $2009 / 10(p<0.001)$. Caries reductions were seen in all socioeconomic groups. This study demonstrates that it is possible to impact upon the prevalence and morbidity of dental caries across the socioeconomic spectrum in a population. The dental health of young children in the NHS Greater Glasgow and Clyde Board area has improved significantly in recent years. 